\section{Lysogeny versus Lysis}

from our Cell Biology Correspondent

THE infection of a susceptible bacterium by a temperate bacteriophage can result either in the lytic replication of the phage and the death of the host or lysogeny, the integration of the repressed phage genome into that of the host which survives the infection. But what determines in the first few minutes after infection which way the infection will go? The answer to this perennial question, according to Hong, Smith and Ames (Proc. US Nat. Acad. Sci., 68, $2258 ; 1971$ ), is the intracellular concentration of cyclic AMP, that ubiquitous regulatory molecule. They reach this conclusion from investigations of the influence of the physiological state of Salmonella typhimurium on lysogenization by the temperate phage P22.

Mutants of this bacterium which have defective adenylate cyclase (ihe enzyme which catalyses cyclic AMP production) or defective cyclic AMP receptor protein (the protein which when active binds cyclic AMP and activates RNA polymerase) are lysogenized by $\mathrm{P} 22$ at considerably lower frequencies than wild type $S$. typhimurium. This is also true of rif mutants of this bacterium which have an altered $\beta$ subunit of RNA polymerase. Hong et al. suggest that it is no coincidence that these three classes of mutants not only reduce the activity of RNA polymerase but also cause the infecting P22 phage to replicate and lyse the cells rather than to lysogenize them.

They believe that in wild type cells cyclic AMP, by binding to the cyclic AMP receptor protein, activates wild type RNA polymerase to transcribe the phage genes, the expression of which establishes lysogeny. The amount of cyclic AMP in the cells will determine between lysis and lysogeny. Because of catabolite repression when there is an abundant supply of energy and little cyclic AMP, lytic replication will occur, but when energy is in short supply and there is a high concentration of cyclic AMP, lysogeny will predominate. The selective advantage of such a regulatory system to the phage is obvious and, as Hong et al. comment, other groups have made, and are about to publish, similar observations and conclusions made with other host and phage combinations; cyclic AMP is therefore probably the deciding factor in all infections by temperate bacteriophages.

In the same issue of the Proceedings (ibid., 2185, 2190), Reichardt and Kaiser and Echols and Green describe in detail the genetic elements that regulate the synthesis of $\lambda$ phage repressor protein which is required to establish and maintain lysogeny of Escherichia coli. The story that emerges from their work is that there are two pathways leading to the synthesis of $\lambda$ repressor which is specified by the $C I$ gene. This gene apparently has two promoter sites prm (promoter for repressor maintenance) and pre (promoter for repressor establishment). Two proteins specified by the genes $C I I$ and $C I I I$ activate the pre promoter during the establishment of lysogeny by binding to a DNA site $c Y$. Mutants of genes CII or CIII of site $c Y$ prevent the transcription of gene $C I$ and hence the synthesis of repressor and the establishment of lysogeny.

Once repressor has been made with wild type $\lambda$ phage using the pre promoter and lysogeny has been established, the smaller amounts of repressor required to be made continuously to main- tain the lysogenic state are transcribed from the prm promoter which becomes active when repressor molecules made from the pre promoter bind to an operator site $\mathrm{Or}$. The pre promoter activated by $C I I$ and $C I I I$ gene proteins is more efficient, in the sense that it allows greater amounts of repressor to be made, than the prm promoter activated by repressor itself. This dual pathway therefore allows the phage to make repressor in large quantities early in the infection and so to establish lysogeny and in smaller amounts subsequently. Echols and Green certainly do not exaggerate by saying that "the pattern of regulation for even so simple a creature as phage $\lambda$ can be relatively complex".

\title{
More about Radio Emission from Air Showers
}

RADIO methods of studying extensive air showers are comparatively new: the first announcement that radio pulses had been detected in coincidence with the shower particles was made by Jelley et al. in 1965 (Nature, 205, 327 ; 1965). Several features of the emission now seem to be firmly established. First, the principal cause of the emission is the transverse deflexion of the shower particles by the Earth's magnetic field-the so-called geomagnetic mechanism; and second, the pulses are large enough to be detectable only because of coherence -in other words, because of the superposition in phase of radiation coming from different stages in the shower development. The radiation pattern on the ground-the radio pulse lateral distribution -is essentially a diffraction pattern determined by the longitudinal development of the shower, and a promising way to study the longitudinal development is through the radio emission. A major astrophysical interest at the present time is the chemical identification of the high-energy cosmic ray primary particles that produce extensive showers in the atmosphere, because this has an important bearing on the problem of cosmic ray origins. It seems that a study of the longitudinal development through the radio emission will contribute significantly to the solution of this problem.

It is therefore of more than passing interest to have a thorough understanding of the mechanism by which the radio emission is produced. Geomagnetic deflexion is certainly the dominant process, but other possible mechanisms have been suggested, and it is important to know quantitatively their relative importance. A careful study by Prescott and his colleagues in Calgary (see the next issue of Nature Physical Science) gives a first answer to this question.

The starting point is the knowledge that geomagnetic deflexion is greatest when a shower moves with its axis at right angles to the Earth's magnetic field lines and is zero when the axis and the field lines are parallel. The experimental aim is to plot the radio pulse amplitude as a function of the sine of the angle $\theta_{g}$, between the shower axis and the field lines, keeping other parameters constant. From the graph, the residual amplitude is determined when $\theta_{g}=0$. For a pure geomagnetic mechanism this amplitude would be zero.

The experiment is complicated by the fact that showers with different $\theta_{g}$ usually have different zenith angles as well. So although two showers have the same number of particles at sea level, their total energy can be different, and the shower maxima can be at different distances from the radio antenna. Unless a correction is made for these differences, it is inadmissible to attribute changes in observed radio pulse amplitude entirely to $\theta_{\mathrm{g}}$. Prescott and his collaborators consider this problem in detail and use other experimental information to determine the necessary corrections. They also make allowance for the background noise level, subtracting this quadratically from the measured amplitude to obtain the true amplitude of the radio pulse.

The measurements were made at a frequency of $22 \mathrm{MHz}$ on showers of size $\sim 10^{6}$ particles, or shower energy $\sim 10^{16} \mathrm{eV}$. A residual pulse height was found at $\theta_{g}=0$ of amplitude $14 \pm 6$ per cent of the amplitude when $\sin \theta_{\mathrm{g}}=1$. This component of the radio emission cannot be attributable to geomagnetic deflexion, and Prescott et al. speculate that it may possibly be caused by the quasi-Cerenkov charge excess mechanism which was one of the first mechanisms to be considered as the source of the radio emission. 